\title{
Three Dimensional MHD Simulations of Parker Instability in Differentially Rotating Disk
}

\author{
T. Matsuzaki, R. Matsumoto ${ }^{4}$ \\ Department of Physics, Faculty of Science, Chiba University, 1-33 \\ Yayoi-Cho, Inage-Ku, Chiba 263, Japan
}

T. Tajima

Institute for Fusion Studies, University of Texas at Austin, Austin, TX 78712, USA

K. Shibata

\section{National Astronomical Obserbatory, Mitaka, Tokyo 181, Japan}

\begin{abstract}
Nonlinear growth of the Parker instability (PI) and the Balbus \& Hawley instability (BHI) in accretion disks have been studied by local three-dimensional magnetohydrodynamic (MHD) simulations. In high- $\beta$ disks $\left(\beta=P_{g a s} / P_{m a g}>1\right)$, the PI has only minor effects on the saturation level of BHI. In low $\beta$ disks $(\beta \leq 1)$, the disk stays in a low- $\beta$ state because magnetic flux cannot escape fast enough to convert the disk into a high- $\beta$ state. We found that even in low- $\beta$ disk the BHI generates fluctuating magnetic fields. The effective magnetic viscosity $\alpha_{B}\left(=-\left\langle B_{r} B_{\phi} / 4 \pi P_{0}\right\rangle\right)$ is $O(0.1)$ when $\beta \sim 1$.
\end{abstract}

It has been thought that the Parker instability sets a lower limit for the plasma $\beta$ in accretion disks because magnetic flux escapes from the disk by buoyancy. In differentially rotating disks, however, the rotation and velocity shear have stabilizing effects on the Parker instability. Meanwhile, fluctuating magnetic fields are generated and amplified by the Balbus \& Hawley instability.

Recentry, several groups have carried out local 3D MHD simulations of the BHI in weakly magnetized disks. They showed that the value of $\alpha_{B}$ in the saturation stage of the instability is $O(0.01)$ when the magnetic field does not have a net vertical flux. Brandenburg et al. (1995) and Stone, Gammie, Hawley \& Balbus (1996) carried out 3D MHD simulations including vertical gravity(i.e., stratification and buoyancy). The size of their simulation box, however, is not large enough to allow the non-linear growth of the PI.

We carried out 3D local MHD simulations of accretion disks by using larger simulation box in both vertical and azimuthal directions. In a frame rotating with the disk with angular speed $\Omega$, we take local Cartesian coordinates with $x, y, z$ in the radial, azimuthal, and vertical direction, respectively. The vertical gravity is assumed to be $g(z)=-G M z /\left(r_{0}^{2}+z^{2}\right)^{3 / 2}$ where $r_{0}$ is the radial

\footnotetext{
${ }^{4}$ Advanced Science Research Center, JAERI, Naka, Japan
} 

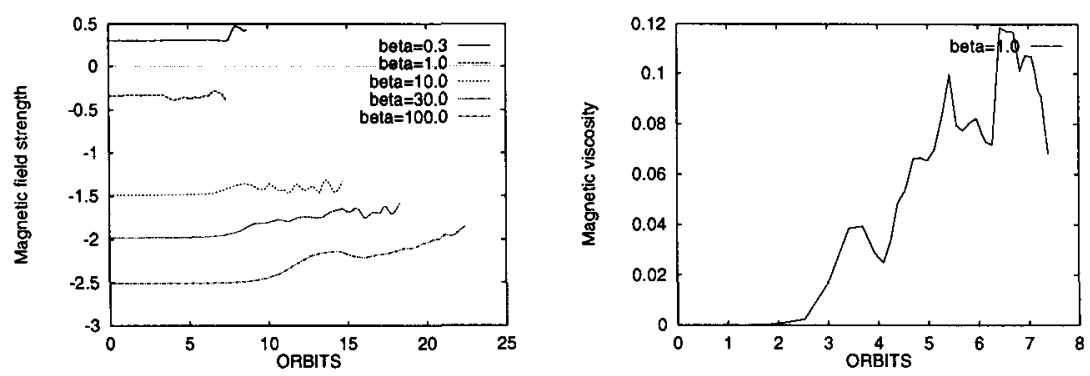

Figure 1. (a) Time evolution of mean square magnetic field strength.

(b) Time evolution of effective magnetic viscosity

distance from the gravitating center. The initial state consists of an isothermal gas layer in magnetohydrostatic equilibrium with horizontal magnetic field. The plasma $\beta$ at $t=0$ is assumed to be uniform. The velocity shear is given by $v_{y}=$ $-1.5 \Omega_{k} x$ where $\Omega_{k}$ is the Keplerian angular speed. We use the normalization $\rho(z=0)=c_{s}=\Omega_{k}=1$, where $c_{s}$ is the sound speed.

A characteristic scale height is defined as $H=c_{s} / \Omega_{k}=1$. The size of the simulation box is $\left(L_{x}, L_{y}, L_{z}\right)=(0.5 H, 18 H, 8 H)$. We computed both upper and lower half plane. The azimuthal boundaries are periodic. The radial boundaries are treated by using slidng periodic condition (Hawley et al. 1995). The upper and lower boundaries are free boundary where waves can transmit. We solved the MHD equations by a modified Lax-Wendroff scheme with artificial viscosity.

Figure 1(a) shows the time evolution of mean square magnetic field strength $\left\langle B^{2} / 8 \pi P_{0}\right\rangle$ for $\beta=0.3,1.0,10.0,30.0$ and 100.0 . When the initial $\beta$ is large $(\beta \gg 1)$, the magnetic field strength increases and approaches to an universal value $(\sim 0.03)$. It is consistent with the results of Stone et al. (1996). When $\beta \leq 1$, we confirmed our previous expectation (Shibata et al. 1990) that once low- $\beta$ disk is formed, it can stay in low- $\beta$ state. Numerical results show sporadic expansion of magnetic loops, which indicates the growth of PI. Since the growth time of the Parker instability is $3-5 H / v_{A}$ when $\beta \sim 1$, it is comparable to that of the Balbus \& Hawley instability. The couplings between BHI and PI may be important when $\beta \leq 1$.

Figure 1(b) shows the magnetic viscosity $\alpha_{B}$ for $\beta=1$. We found that, even in low- $\beta$ disk, BHI grows and generates fluctuating fields. The value of $\alpha_{B}$ in the saturation stage of the instability is $\simeq 0.1$. This value of $\alpha_{B}$ is an order of magnitude larger than that in high- $\beta$ disk $(\beta \sim 10)$.

\section{References}

Brandenburg et al. 1995, ApJ, 446, 741

Hawley, J. F., Gammie, C. F., \& Balbus, S. A. 1995, ApJ, 440, 742

Parker, E. N. 1966, ApJ, 145, 811

Matsumoto, R., Horiuchi, T., Shibata, K., \& Hanawa, T. 1988 PASJ, 40, 171

Matsumoto, R., \& Tajima, T. 1995, ApJ, 445, 767

Shibata, K., Tajima, T., \& Matsumoto, R. 1990, ApJ, 350, 295

Stone et al. 1996, ApJ, 463, 656 